\title{
Morphological Changes of The Tricuspid Valve Complex in Functional Tricuspid Regurgitation On Contrast-Enhanced Computed Tomography
}

Hiroki Uchiyama ( $\nabla$ hirouchiyama.cvs@gmail.com )

Sapporo Medical University https://orcid.org/0000-0002-5867-7810

Kazutoshi Tachibana

Hakodate Goryoukaku Hospital

Koichi Osuda

Hakodate Goryoukaku Hospital

Nobuyoshi Kawaharada

Sapporo Medical University School of Medicine Graduate School of Medicine: Sapporo Ika Daigaku Igakubu Daigakuin Igaku Kenkyuka

Research article

Keywords: Functional tricuspid regurgitation, Contrast-enhanced computed tomography, Tricuspid annulus area, Tricuspid annulus circumference, Right ventricular volume, Distance between papillary muscles

Posted Date: November 17th, 2021

DOI: https://doi.org/10.21203/rs.3.rs-1063092/v1

License: (9) This work is licensed under a Creative Commons Attribution 4.0 International License. Read Full License 


\section{Abstract}

Background: Whether it is possible to perform morphological evaluation of functional tricuspid regurgitation (FTR) on contrast-enhanced computed tomography (CT) was examined by evaluating the relationships between the parameters measured by contrast-enhanced CT and TR severity on transthoracic echocardiography.

Methods: Fifty patients underwent contrast-enhanced CT. Tricuspid annulus area (TAA), tricuspid annulus circumference (TAC), right ventricular volume (RVV), and the distances between the tips and bases of the papillary muscles were measured on contrast-enhanced CT in diastole and systole. Fifty cases were divided into 34 in the TR $\leq$ mild group (none TR: 3 cases, trivial TR: 24 cases, mild TR: 7 cases), and 16 in the TR $\geq$ moderate group (moderate TR: 8 cases, severe TR: 8 cases) using the TR grade measured by transthoracic echocardiography, and then differences between the groups were examined.

Results: Significant differences were found in TAA, TAC, and RVV $(p<0.01)$ and the distances between the tips of the anterior and posterior papillary muscles $(p<0.05)$ in both diastole and systole. Since the septal papillary muscle could not be identified in 18 cases (36.0\%), only the distance between the anterior and posterior papillary muscles was measurable in all cases. On subgroup analysis, risk factors for FTR $\geq$ moderate were evaluated by multiple logistic regression analysis. The risk factors identified in the multivariable risk factor analysis were diastolic RVV (odds ratio 1.083 [95\% confidence interval 1.0291.139]) and systolic RVV (odds ratio 0.951 [95\% confidence interval 0.906-0.997]).

Conclusions: TAA, TAC, RVV, and the distance between the tips of the anterior and posterior papillary muscles measured on contrast-enhanced CT were shown to be significantly increased in the TR $\geq$ moderate group. Detailed morphological assessment of FTR is possible by contrast-enhanced CT.

\section{Background}

The tricuspid valve has long been called "the forgotten valve" or "the silent valve", and its importance has been undervalued compared with left-sided valve diseases. However, the tricuspid valve is composed of leaflets (anterior leaflet, posterior leaflet, and septal leaflet), an annulus, chordae tendineae, papillary muscles (anterior papillary muscle, posterior papillary muscle, septal papillary muscle), right atrium, and right ventricle. Since it is a complicated structure, it has been found that it deeply affects right ventricular function, and that residual or worsening tricuspid regurgitation (TR) also leads to worsening quality of life (QOL) and prognosis [1].

Functional tricuspid regurgitation (FTR) occurs due to morphological changes of the tricuspid valve complex that develop secondary to tricuspid annulus dilation and ventricular enlargement as a result of volume or pressure overload of the right ventricle due to left-sided valve diseases $[2,3,4,5]$.

FTR severity is diagnosed by transthoracic echocardiography (TTE) and estimated semiquantitatively using the range or area of the regurgitant jet. TTE is performed easily, and a past study evaluated 
morphological changes of the tricuspid valve complex by transthoracic echocardiography [6]. However, there are some limitations of transthoracic echocardiography, such as the need for the evaluator to have experience and restriction of ultrasound examination by ribs or air.

On the other hand, contrast-enhanced computed tomography (CT) is performed easily and is useful for morphological assessment. Recently, detailed preoperative morphological assessment using contrastenhanced CT has been increasing, for example, for transcatheter aortic valve implantation (TAVI) $[7,8,9]$. However, few papers have considered morphological assessment of FTR using contrast-enhanced CT.

We postulated that, in FTR, morphological changes of tricuspid annulus area, tricuspid annulus circumference, right ventricular volume, and distance between papillary muscles could be identified by contrast-enhanced CT.

In this study, whether it is possible to perform morphological evaluation of FTR with contrast-enhanced CT was examined by evaluating the relationships between the parameters measured by contrastenhanced CT and TR severity on transthoracic echocardiography.

\section{Methods}

¿Study population

Between April 2018 and April 2020, 50 patients planned for cardiovascular surgery underwent contrastenhanced CT and transthoracic echocardiography. Patients who had de novo myocardial infarction within less than 28 days, unstable angina, end-stage renal failure, infective endocarditis, active hemorrhagic diseases (gastrointestinal bleeding, trauma, etc.), or postoperative pacemaker implantation were excluded. This study was approved by the research ethics committee of Sapporo Medical University.

The 50 cases were divided into two groups, TR $\leq$ mild group and TR $\geq$ moderate group, by TR grade measured on transthoracic echocardiography, and then differences between the groups were examined.

[Contrast-enhanced computed tomography

ECG-gated 320-detector-row multislice computed tomography (Aquilion one, Toshiba Medical Systems, Tokyo, Japan) was used for this study. In order to ensure that the tricuspid annulus and right ventricle would be clearly depicted, very early phase images were taken.

The reconstructed volume data images were transferred to OsiriX (Pixmeo, Geneva, Switzerland) and Ziostation2 (Ziosoft, Tokyo, Japan). Tricuspid annulus area (TAA), tricuspid annulus circumference (TAC), right ventricular volume (RVV), and the distances between the tips ( $t$ ) and bases (b) of the papillary muscles (anterior, posterior, and septal) were measured on contrast-enhanced CT. Each was measured at diastole (d) and at systole (s). Figure 1 shows the measurement chart using OsiriX for the tricuspid annulus area, the perimeter of the tricuspid annulus, and tricuspid annulus circumference, Figure 2 shows 
the measurement chart using Ziostation2 for right ventricular volume, and Figure 3 shows the measurement chart using OsiriX for the distances between the tips and bases of the papillary muscles.

पTransthoracic echocardiography

The Philips iE33 (Koninklijke Philips N.V., Amsterdam, Netherlands) was used in this study. In addition to the general transthoracic echocardiographic measurements (ejection fraction (EF), aortic diameter (AOD), left atrial diameter (LAD), left ventricular end diastolic diameter (LVDd), left ventricular end systolic diameter (LVDs), interventricular septal thickness at end diastole (IVSTD), posterior wall thickness at end diastole (PWTD), end diastolic volume (EDV), end systolic volume (ESV)), tricuspid regurgitation grade, tricuspid annulus diameter (TA, end-diastole, 4-chamber view), vena contracta (VC), jet area (JA), tricuspid annulus plane systolic excursion (TAPSE), functional area change (FAC), predicted right ventricular pressure (RVP), and inferior vena cava diameter (IVC) were also measured. The prevalence of atrial fibrillation was evaluated, and the severity of other valvular diseases was evaluated as 0 : none, 1: trivial, 2: mild, 3: moderate, and 4: severe.

¿Statistical analysis

The two-sample $t$-test was used to test the mean differences between the two groups, and $p<0.05$ was considered significant. Furthermore, multiple logistic regression analysis was performed on the two groups, and the risk factors contributing to FTR $\geq$ moderate were examined. The dependent variable was FTR $\geq$ moderate or not, and the independent variables were diastolic and systolic tricuspid annulus areas, tricuspid annulus circumference, right ventricular volume, and the distances between the tips and bases of the anterior and posterior papillary muscles, which were measurable in all cases. Univariate risk factor analysis and multivariate risk factor analysis were performed. On multivariate risk factor analysis, the risk factor was selected by the variable increase method (likelihood ratio) using the independent variables with $p<0.05$ on the univariate risk factor analysis. All statistical analyses were performed using IBM SPSS Statistics version 26.0 (IBM, Armonk, NY, USA).

\section{Results}

Table 1 shows the patients' characteristics and the parameters of transthoracic echocardiography for each TR grade. Of the 50 cases, 34 were in the TR $\leq$ mild group (none TR: 3 cases, trivial TR: 24 cases, mild TR: 7 cases), and 16 were in the TR $\geq$ moderate group (moderate TR: 8 cases, severe TR: 8 cases). There were no significant differences in age, sex, or body surface area between the two groups. Significant differences were observed in LAD, IVSTD, MR severity, and PR severity in the parameters of transthoracic echocardiography.

Table 2 shows tricuspid annulus area (TAA), tricuspid annulus circumference (TAC), right ventricular volume (RVV), and the distances between the papillary muscles (anterior, posterior, and septal) measured on contrast-enhanced CT. Significant differences were found in tricuspid annulus area (TAA), tricuspid annulus circumference (TAC), and right ventricular volume (RVV) in both diastole and systole $(p<0.01)$. As 
for the distances between papillary muscles, the anterior and posterior papillary muscles could be identified on contrast-enhanced CT in all cases, but the septal papillary muscle could not be identified in 18 cases (36.0\%); therefore, only the distances between the anterior and posterior papillary muscles were measurable in all cases. There were significant differences between groups in the diastolic and systolic distances between the tips of the anterior and posterior papillary muscles (dtAP and stAP), diastolic distance between the tips of the posterior and septal papillary muscles (dtPS), and the diastolic and systolic distances between the bases of the posterior and septal papillary muscles (dbPS and sbPS)

$(p<0.05)$. Box-whisker plots and $p$-values for the two groups are shown (tricuspid annulus area in Figure 4, tricuspid annulus circumference in Figure 5, right ventricular volume in Figure 6, distances between the tips of the anterior and posterior papillary muscles in Figure 7, and distances between the bases of the anterior and posterior papillary muscles in Figure 8).

In addition, as a subgroup analysis, multiple logistic regression analysis was performed on two groups to identify the risk factor most strong related to FTR $\geq$ moderate. The univariate risk factor analysis is shown in Table 3, and the multivariate risk factor analysis is shown in Table 4. Univariate risk factor analysis showed that tricuspid annulus area, tricuspid annulus circumference, right ventricular volume, and the distance between the tips of the anterior and posterior papillary muscles had $p<0.05$ in both diastole and systole. On multivariate risk factor analysis, the risk factors were selected by the variable increase method (likelihood ratio) using the independent variables with $p<0.05$ on the univariate risk factor analysis, and the risk factors were diastolic right ventricular volume (dRVV)(odds ratio 1.083 [95\% confidence interval 1.029-1.139]) and systolic right ventricular volume (sRVV) (odds ratio 0.951 [95\% confidence interval 0.906-0.997]).

\section{Discussion}

The tricuspid annulus area (TAA), tricuspid annulus circumference (TAC), and right ventricular volume (RVV) measured on contrast-enhanced CT were significantly increased in the TR $\geq$ moderate group, showing that tricuspid annulus dilation and ventricular enlargement are causes of FTR progression. This corroborates the relationships between contrast-enhanced CT parameters and TR grade measured by transthoracic echocardiography. According to the 2017 ESC/EACTS guidelines, tricuspid valve surgery for secondary TR is class I for severe TR undergoing left-sided valve surgery, and class lla for mild or moderate TR with a dilated annulus ( $\geq 40 \mathrm{~mm}$ or $>21 \mathrm{~mm} / \mathrm{m}^{2}$ on $2 \mathrm{D}$ echocardiography) undergoing leftsided valve surgery [10]; in the present study, moderate and severe TR significantly increased tricuspid annulus area (TAA), tricuspid annulus circumference (TAC), and right ventricular volume (RVV) on contrast-enhanced CT. Therefore, the parameters of contrast-enhanced CT may also be considered in the indications for FTR surgery in the future. In general, tricuspid annulus diameter is measured in enddiastole by transthoracic echocardiography, but since tricuspid regurgitation occurs during systole, one study pointed out that the tricuspid annulus diameter should be measured in systole [11], with which we agree. Measurement of tricuspid annulus diameter and determination of ring size of the tricuspid valve should be done in systole. 
Regarding the distance between papillary muscles, a significant difference was observed in the distance between the tips of the anterior and posterior papillary muscles, and it is considered that it tends to increase as FTR becomes more severe. In about 1/3 (36.0\%) of cases, the septal papillary muscle could not be identified on contrast-enhanced CT. According to autopsy reports, cases with many small septal papillary muscles or tendinous cords that appear directly from the septal walls of the right ventricle have been described, and there are many variations of septal papillary muscles [12]. The findings of the present study were similar, and there were many cases in which septal muscles could not be identified because of the many variations. Anterior and posterior papillary muscles were identified in all cases; only the distances between the anterior and posterior papillary muscles could be measured effectively. In some cases, the posterior papillary muscle was also small, and only the anterior papillary muscle was clearly visible, so it is thought that the anterior papillary muscle contributes most to the tricuspid valve.

Multiple logistic regression analysis suggested that diastolic and systolic right ventricular volumes (dRVV and SRVV) contributed to FTR $\geq$ moderate. The odds ratio was 1.083 for diastolic right ventricular volume (dRVV) and 0.951 for systolic right ventricular volume (sRVV), which cannot be said to be large, but since the right ventricular volume also depends on whole body volume, volume management is important for TR management.

Kabasawa and colleagues also evaluated FTR on contrast-enhanced CT [13] in 35 patients who underwent contrast-enhanced CT, and end-diastolic and end-systolic tricuspid valve annular diameters (TVADs), tethering angles, and tethering height were significantly correlated with preoperative TR severity. The result for the tricuspid annulus was similar to that of the present study, and the present study did not evaluate tethering angles and tethering height, but evaluated right ventricular volume and the distances between the papillary muscles.

In comparison with ultrasonography, contrast-enhanced CT is slightly more invasive because of the use of contrast media and radiation exposure. However, it is convenient, because it can provide morphological evaluations, especially quantitative measurements, easily and in detail. In the present study, the detailed morphological assessment of FTR was possible using contrast-enhanced CT.

In recent years, minimally invasive transcatheter approaches for TR have been introduced, such as the Mitraclip for TR and the FORMA tricuspid repair system as leaflet devices and the Trialign percutaneous tricuspid valve annuloplasty system, the Cardioband tricuspid annuloplasty system, and the TriCinch tricuspid valve repair as annuloplasty devices. Another examples are Transcatheter Tricuspid Valve Replacement (TTVR) and Caval Valve Implantation (CAVI) [14]. In preoperative planning for tricuspid valve treatment in the future, it will be essential to evaluate the tricuspid valve complex on contrastenhanced CT. There are reports that CT provides very useful information on annular structure and dimensions, the quality and amount of annular tissue, and its relationship with the right coronary artery [15]

There are several limitations to this study. The tricuspid annulus actually has three-dimensional geometry $[16,17]$. However, in the present study, because the tricuspid annulus was measured in a contrast- 
enhanced CT section, the true annulus structure may not have been evaluated.

There were significantly more cases of left atrial enlargement, mitral regurgitation, and pulmonary regurgitation in the FTR $\geq$ moderate group. It has been reported that left atrial enlargement and atrial fibrillation are risk factors for postoperative TR deterioration [18]. In the present study, atrial fibrillation cases showed no significant difference, but the prevalence of mitral valve disease, pulmonary valve disease, or pulmonary hypertension may also be a confounding factor.

The present study did not evaluate the same cases over time, but if they were to be classified according to the surgical intervention and evaluated over time, we may be able to further deepen our understanding of the morphological changes in FTR and the surgical indications.

\section{Conclusions}

Tricuspid annulus area, tricuspid annulus circumference, right ventricular volume, and the distances between the tips of the anterior and posterior papillary muscles measured on contrast-enhanced CT were shown to be significantly increased in the $T R \geq$ moderate group.

Detailed morphological assessment of functional tricuspid regurgitation is possible on contrastenhanced CT.

\section{Abbreviations}

TR: Tricuspid regurgitation; FTR: Functional tricuspid regurgitation; TTE: Transthoracic echocardiography; CT: Computed tomography; TAA: Tricuspid annulus area; TAC: Tricuspid annulus circumference; RVV: Right ventricular volume; AP: Between the anterior and posterior papillary muscles; PS: Between the posterior and septal papillary muscles; SA: Between the septal and anterior papillary muscles; EF: Ejection fraction; AOD: Aortic diameter; LAD: Left atrial diameter; LVDd: Left ventricular end diastolic diameter; LVDs: Left ventricular end systolic diameter; IVSTD: Interventricular septal thickness at end diastole; PWTD: Posterior wall thickness at end diastole; EDV: End diastolic volume; ESV: End systolic volume; TA: Tricuspid annulus diameter; VC: Vena contracta; JA: Jet area; TAPSE: Tricuspid annulus plane systolic excursion; FAC: Functional area change; RVP: Right ventricular pressure; RAP: Right atrial pressure; IVC: Inferior vena cava diameter; AR: Aortic regurgitation; AS: Aortic stenosis; MR: Mitral regurgitation; MS: Mitral stenosis; PR: Pulmonary regurgitation; PS: Pulmonary stenosis

\section{Declarations}

\section{Acknowledgements}

Not applicable.

\section{Authors' contributions}


$\mathrm{HU}$ designed the study and wrote the initial draft of the manuscript. KO collected the data. KT and NK contributed to analysis and interpretation of data, and assisted in the preparation of the manuscript. All authors read and approved the final manuscript.

\section{Funding}

No funding was received for this work.

\section{Availability of data and materials}

The datasets used and/or analysed during the current study are available from the corresponding author on reasonable request.

\section{Ethics approval and consent to participate}

Not applicable.

\section{Consent for publication}

Not applicable.

\section{Competing interests}

The authors declare that they have no competing interests.

\section{Author details}

${ }^{1}$ Department of Cardiovascular Surgery, Sapporo Medical University, Sapporo, Japan; ${ }^{2}$ Department of Cardiovascular Surgery, Hakodate Goryoukaku Hospital, Hakodate, Japan; ${ }^{3}$ Division of Radiology, Hakodate Goryoukaku Hospital, Hakodate, Japan

\section{References}

[1] El-Eshmawi A, Pandis D, Adams DH, Tang GH. Tricuspid valve surgery: repair and replacement. Minerva Cardioangiol. 2018;66:700-12.

[2] Tei C, Pilgrim JP, Shah PM, Ormiston JA, Wong M. The tricuspid valve annulus: study of size and motion in normal subjects and in patients with tricuspid regurgitation. Circulation. 1982;66:665-71.

[3] Ubago JL, Figueroa A, Ochoteco A, Colman T, Duran RM, Duran CG. Analysis of the amount of tricuspid valve annular dilatation required to produce functional tricuspid regurgitation. Am J Cardiol. 1983;52:155-8.

[4] Sagie A, Schwammenthal E, Padial LR, Vazquez de Prada JA, Weyman AE, Levine RA. Determinants of functional tricuspid regurgitation in incomplete tricuspid valve closure: doppler color flow study of 109 
patients. J Am Coll Cardiol. 1994;24:446-53.

[5] Dreyfus GD, Corbi PJ, Chan KM, Bahrami T. Secondary tricuspid regurgi- tation or dilatation: which should be the criteria for surgical repair? Ann Thorac Surg. 2005;79:127-32.

[6] Spinner EM, Lerakis S, Higginson J, Pernetz M, Howell S, Veledar E et al. Correlates of tricuspid regurgitation as determined by 3D echocardiography: pulmonary arterial pressure, ventricle geometry, annular dilatation, and papillary muscle displacement. Circ Cardiovasc Imaging. 2012;5:43-50.

[7] Bloomfield GS, Gillam LD, Hahn RT, Kapadia S, Leipsic J, Lerakis S et al. A practical guide to multimodality imaging of transcatheter aortic valve replacement. J Am Coll Cardiol Img. 2012;5:441-55.

[8] Ribeiro HB, Webb JG, Makkar RR, Cohen MG, Kapadia SR, Kodali S et al. Predictive factors, management, and clinical outcomes of coronary obstruction following transcatheter aortic valve implantation. J Am Coll Cardiol. 2013;62:1552-62.

[9] Latsios G, Spyridopoulos TN, Toutouzas K, Synetos A, Trantalis G, Stathogiannis K et al. Multi-slice CT (MSCT) imaging in pretrans-catheter aortic valve implantation (TAVI) screening. How to perform and how to interpret. Hellenic Journal of Cardiology. 2018;59:3-7.

[10] Baumgartner H, Falk V, Bax JJ, Bonis MD, Hamm C, Holm PJ et al. 2017 ESC/EACTS Guidelines for the management of valvular heart disease. European Heart Journal. 2017;38:2739-91.

[11] Calafiore A, laco AL, Romeo A, Scandura S, Meduri R, Varone E. Echocardiographic-based treatment of functional tricuspid regurgitation. J Thorac Cardiovasc Surg. 2011;142:308-13.

[12] Silver MD, Lam JHC, Ranganathan N, Wigle ED. Morphology of the human tricuspid valve. Circulation. 1971;43:333-48.

[13] Kabasawa M, Kohno H, Ishizaka T, Ishida K, Funabashi N, Kataoka A et al. Assessment of functional tricuspid regurgitation using 320-detector-row multislice computed tomography: Risk factor analysis for recurrent regurgitation after tricuspid annuloplasty. Thorac Cardiovasc Surg. 2014;147:312-20.

[14] Moscarella E, Brugaletta S, Sabaté M. New percutaneous interventions in heart failure. Minerva Cardioangiol. 2019;67:145-62.

[15] Ancona F, Stella S, Capogrosso C, Marini C, Fisicaro A, Margonato A et al. Tricuspid valve imaging. Minerva Cardioangiol. 2018;66:680-90.

[16] Fukuda S, Saracino G, Matsumura Y, Daimon M, Tran H, Greenberg NL et al. Three-dimensional geometry of the tricuspid annulus in healthy subjects and in patients with functional tricuspid regurgitation: a real-time, 3-dimensional echocardio- graphic study. Circulation. 2006;114(suppl):I-492-I498. 
[17] Matsuyama K, Matsumoto M, Sugita T, Nishizawa J, Tokuda Y, Matsuo T. Predictors of residual tricuspid regurgitation after mitral valve surgery. Ann Thorac Surg. 2003;75:1826-8.

[18] Rogers JH, Bolling SF. The Tricuspid Valve Current Perspective and Evolving Management of Tricuspid Regurgitation. Circulation. 2009;119:2718-25

\section{Tables}

Table 1: Patients' characteristics and parameters measured by transthoracic echocardiography 


$\begin{array}{llll}\text { All cases } & \text { TR } \leq \text { mild } & \text { TR } \geq \text { moderate } & \text { P-value } \\ (n=50) & (n=34) & (n=16)\end{array}$

\section{Patients' characteristics}

\begin{tabular}{lllll} 
Age $(\mathrm{y})$ & $73.6 \pm 9.2$ & $72.3 \pm 10.2$ & $76.5 \pm 5.7$ & 0.066 \\
\hline Male sex, $\mathrm{n}(\%)$ & $31(62.0)$ & $22(64.7)$ & $9(56.3)$ & 0.575 \\
\hline BSA $\left(\mathrm{cm}^{2}\right)$ & $1.59 \pm 0.16$ & $1.60 \pm 0.15$ & $1.57 \pm 0.19$ & 0.569
\end{tabular}

\section{Transthoracic echocardiography}

\begin{tabular}{lllll}
\hline EF $(\%)$ & $64.3 \pm 10.1$ & $63.5 \pm 10.8$ & $65.9 \pm 8.6$ & 0.421 \\
\hline AOD $(\mathrm{mm})$ & $33.7 \pm 5.2$ & $33.9 \pm 5.4$ & $33.3 \pm 5.1$ & 0.702 \\
\hline LAD $(\mathrm{mm})$ & $43.3 \pm 9.2$ & $40.7 \pm 8.0$ & $48.9 \pm 9.4$ & 0.003 \\
\hline LVDd $(\mathrm{mm})$ & $48.3 \pm 7.5$ & $48.2 \pm 7.7$ & $48.4 \pm 7.2$ & 0.943 \\
\hline LVDs $(\mathrm{mm})$ & $31.8 \pm 9.2$ & $32.5 \pm 9.1$ & $30.4 \pm 9.6$ & 0.456 \\
\hline IVSTD (mm) & $10.6 \pm 1.9$ & $11.1 \pm 2.0$ & $9.6 \pm 1.4$ & 0.013 \\
\hline PWTD (mm) & $10.2 \pm 1.7$ & $10.5 \pm 1.7$ & $9.5 \pm 1.5$ & 0.060 \\
\hline EDV $(\mathrm{mL})$ & $99.9 \pm 36.6$ & $106.5 \pm 37.1$ & $86.4 \pm 32.3$ & 0.071 \\
\hline ESV $(\mathrm{mL})$ & $37.7 \pm 23.6$ & $41.5 \pm 26.5$ & $29.7 \pm 13.3$ & 0.099 \\
\hline Af rhythm, n (\%) & $11(22.0)$ & $5(14.7)$ & $6(37.5)$ & 0.116 \\
\hline Asynergy, n $(\%)$ & $4(8.0)$ & $1(2.9)$ & $3(18.8)$ & 0.15 \\
\hline TA $(\mathrm{mm})$ & $31.8 \pm 6.1$ & $29.1 \pm 4.0$ & $36.0 \pm 6.4$ & $<0.001$ \\
\hline VC $(\mathrm{mm})$ & $5.8 \pm 4.2$ & $3.4 \pm 1.0$ & $8.1 \pm 4.8$ & 0.004 \\
\hline JA (cm $\left.{ }^{2}\right)$ & $6.0 \pm 6.3$ & $1.7 \pm 0.9$ & $10.0 \pm 6.6$ & $<0.001$ \\
\hline TAPSE & $19.9 \pm 3.7$ & $20.5 \pm 3.6$ & $19.0 \pm 3.8$ & 0.188 \\
\hline FAC $(\%)$ & $38.6 \pm 7.2$ & $39.5 \pm 4.8$ & $37.0 \pm 10.7$ & 0.472 \\
\hline RVP (mmHg) & $30.7 \pm 10.9$ & $25.9 \pm 6.7$ & $41.6 \pm 10.7$ & $<0.001$ \\
\hline RAP (mmHg) & $6.5 \pm 3.4$ & $5.3 \pm 1.8$ & $9.2 \pm 4.7$ & 0.016 \\
\hline IVC (mm) & $16.4 \pm 4.6$ & $15.0 \pm 3.2$ & $19.5 \pm 5.7$ & 0.008 \\
\hline AR & $1.4 \pm 1.4$ & $1.4 \pm 1.4$ & $1.4 \pm 1.3$ & 0.841 \\
\hline AS & $0.5 \pm 1.2$ & $0.6 \pm 1.4$ & $0.2 \pm 0.4$ & 0.163 \\
\hline MR & $2.0 \pm 1.3$ & $1.7 \pm 1.3$ & $2.6 \pm 1.2$ & 0.036 \\
\hline
\end{tabular}




\begin{tabular}{lllll} 
MS & $0.08 \pm 0.6$ & $0.0 \pm 0.0$ & $0.25 \pm 1.0$ & 0.333 \\
\hline PR & $1.2 \pm 0.6$ & $1.0 \pm 0.4$ & $1.6 \pm 0.9$ & 0.034 \\
\hline PS & $0.0 \pm 0.0$ & $0.0 \pm 0.0$ & $0.0 \pm 0.0$ & -
\end{tabular}

EF: ejection fraction

AOD: aortic diameter

LAD: left atrial diameter

LVDd: left ventricular end diastolic diameter

LVDs: left ventricular end systolic diameter

IVSTD: interventricular septal thickness at end diastole

PWTD: posterior wall thickness at end diastole

EDV: end diastolic volume

ESV: end systolic volume

TA: tricuspid annulus diameter (4 chamber view, diastolic)

VC: vena contracta

JA: jet area

TAPSE: tricuspid annulus plane systolic excursion

FAC: functional area change

RVP: right ventricular pressure

RAP: right atrial pressure

IVC: inferior vena cava diameter

AR: aortic regurgitation

AS: aortic stenosis

MR: mitral regurgitation

MS: mitral stenosis 
PR: pulmonary regurgitation

PS: pulmonary stenosis

Table 2: Parameters measured on contrast-enhanced CT

$\begin{array}{llll}\text { All cases } & \text { TR } \leq \text { mild } & \text { TR } \geq \text { moderate } & \text { P-value } \\ (n=50) & (n=34) & (n=16)\end{array}$

Contrastenhanced CT

\begin{tabular}{|lllll}
\hline d TAA $\left(\mathrm{cm}^{2}\right)$ & $14.1 \pm 4.2$ & $12.3 \pm 2.3$ & $17.7 \pm 5.1$ & 0.001 \\
\hline s TAA $\left(\mathrm{cm}^{2}\right)$ & $12.3 \pm 4.2$ & $10.4 \pm 2.4$ & $16.2 \pm 4.5$ & $<0.001$ \\
\hline d TAC $(\mathrm{mm})$ & $136.5 \pm 18.3$ & $129.6 \pm 11.5$ & $151.3 \pm 21.3$ & 0.001 \\
\hline s TAC $(\mathrm{mm})$ & $126.9 \pm 20.2$ & $118.3 \pm 13.2$ & $145.2 \pm 20.6$ & $<0.001$ \\
\hline d RW $(\mathrm{mL})$ & $161.5 \pm 75.4$ & $127.4 \pm 35.4$ & $233.8 \pm 87.3$ & $<0.001$ \\
\hline s RVV $(\mathrm{mL})$ & $106.0 \pm 56.8$ & $86.8 \pm 30.1$ & $146.7 \pm 77.1$ & 0.008 \\
\hline d t AP $(\mathrm{mm})$ & $25.8 \pm 5.3$ & $24.5 \pm 4.3$ & $28.7 \pm 6.1$ & 0.007 \\
\hline s t AP $(\mathrm{mm})$ & $19.9 \pm 4.8$ & $18.8 \pm 4.5$ & $22.4 \pm 4.6$ & 0.012 \\
\hline d b AP $(\mathrm{mm})$ & $29.6 \pm 7.2$ & $28.4 \pm 5.9$ & $32.1 \pm 9.2$ & 0.090 \\
\hline s b AP $(\mathrm{mm})$ & $24.3 \pm 6.2$ & $23.6 \pm 5.5$ & $25.9 \pm 7.4$ & 0.227 \\
\hline d t PS $(\mathrm{mm})$ & $20.9 \pm 7.1$ & $17.8 \pm 3.3$ & $25.4 \pm 8.7$ & 0.007 \\
\hline s t PS $(\mathrm{mm})$ & $16.3 \pm 6.7$ & $14.1 \pm 3.0$ & $19.4 \pm 9.2$ & 0.061 \\
\hline d b PS $(\mathrm{mm})$ & $26.4 \pm 10.0$ & $22.5 \pm 6.2$ & $32.1 \pm 11.9$ & 0.004 \\
\hline s b PS $(\mathrm{mm})$ & $21.4 \pm 7.3$ & $18.7 \pm 4.6$ & $25.2 \pm 8.9$ & 0.011 \\
\hline d t SA $(\mathrm{mm})$ & $32.3 \pm 5.1$ & $31.3 \pm 4.1$ & $33.8 \pm 6.1$ & 0.154 \\
\hline s t SA $(\mathrm{mm})$ & $26.1 \pm 4.9$ & $25.4 \pm 3.7$ & $27.0 \pm 6.2$ & 0.430 \\
\hline d b SA $(\mathrm{mm})$ & $35.7 \pm 8.0$ & $34.2 \pm 6.6$ & $37.9 \pm 9.6$ & 0.219 \\
\hline s b SA $(\mathrm{mm})$ & $28.2 \pm 6.1$ & $28.1 \pm 5.1$ & $28.2 \pm 7.6$ & 0.966 \\
\hline
\end{tabular}

d TAA: diastolic tricuspid annulus area

s TAA: systolic tricuspid annulus area

d TAC: diastolic tricuspid annulus circumference 
S TAC: systolic tricuspid annulus circumference

d RVV: diastolic right ventricular volume

s RVV: systolic right ventricular volume

d t AP: diastolic distance between the tips of the anterior and posterior papillary muscles st AP: systolic distance between the tips of the anterior and posterior papillary muscles d b AP: diastolic distance between the bases of the anterior and posterior papillary muscles s b AP: systolic distance between the bases of the anterior and posterior papillary muscles $\mathrm{d}$ t PS: diastolic distance between the tips of the posterior and septal papillary muscles s t PS: systolic distance between the tips of the posterior and septal papillary muscles $\mathrm{d} b$ PS: diastolic distance between the bases of the posterior and septal papillary muscles s b PS: systolic distance between the bases of the posterior and septal papillary muscles d t SA: diastolic distance between the tips of the septal and anterior papillary muscles s t SA: systolic distance between the tips of the septal and anterior papillary muscles d b SA: diastolic distance between the bases of the septal and anterior papillary muscles s b SA: systolic distance between the bases of the septal and anterior papillary muscles Table 3: Univariable risk factor analysis for FTR $\geq$ moderate 


\begin{tabular}{llll} 
Variable & Odds ratio & $95 \% \mathrm{Cl}$ & P-value \\
\hline d TAA $\left(\mathrm{cm}^{2}\right)$ & 1.548 & $1.194-2.008$ & 0.001 \\
\hline s TAA $\left(\mathrm{cm}^{2}\right)$ & 1.622 & $1.233-2.135$ & 0.001 \\
\hline d TAC $(\mathrm{mm})$ & 1.092 & $1.035-1.152$ & 0.001 \\
\hline s TAC $(\mathrm{mm})$ & 1.102 & $1.042-1.166$ & 0.001 \\
\hline d RW $(\mathrm{mL})$ & 1.042 & $1.015-1.070$ & 0.002 \\
\hline s RW $(\mathrm{mL})$ & 1.027 & $1.008-1.048$ & 0.006 \\
\hline d t AP $(\mathrm{mm})$ & 1.183 & $1.036-1.350$ & 0.013 \\
\hline s t AP $(\mathrm{mm})$ & 1.193 & $1.029-1.382$ & 0.019 \\
\hline d b AP $(\mathrm{mm})$ & 1.078 & $0.986-1.179$ & 0.098 \\
\hline s b AP $(\mathrm{mm})$ & 1.063 & $0.963-1.175$ & 0.226
\end{tabular}

d TAA: diastolic tricuspid annulus area

s TAA: systolic tricuspid annulus area

d TAC: diastolic tricuspid annulus circumference

S TAC: systolic tricuspid annulus circumference

d RVV: diastolic right ventricular volume

s RVV: systolic right ventricular volume

d t AP: diastolic distance between the tips of the anterior and posterior papillary muscles

s t AP: systolic distance between the tips of the anterior and posterior papillary muscles

$\mathrm{d} b$ AP: diastolic distance between the bases of the anterior and posterior papillary muscles s b AP: systolic distance between the bases of the anterior and posterior papillary muscles Table 4: Multivariable risk factor analysis for FTR $\geq$ moderate

\begin{tabular}{|clll} 
Variable & Odds ratio & $95 \% \mathrm{Cl}$ & P-value \\
\hline d RVV $(\mathrm{mL})$ & 1.083 & $1.029-1.139$ & 0.020 \\
\hline $\mathbf{s ~ R V V}(\mathrm{mL})$ & 0.951 & $0.906-0.997$ & 0.037 \\
\hline
\end{tabular}

d RVV: diastolic right ventricular volume 


\section{Figures}

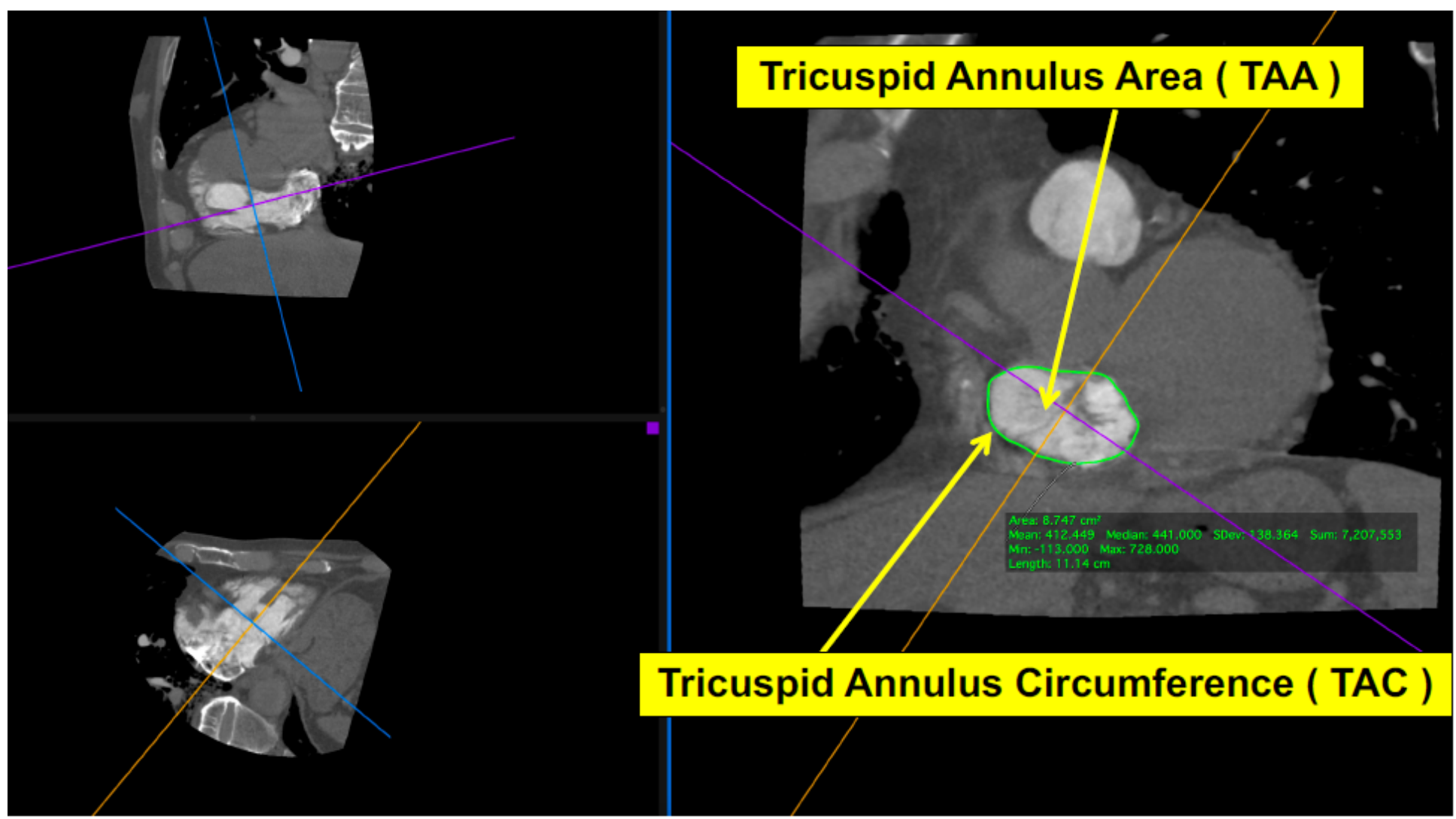

Figure 1

Tricuspid annulus area (TAA) and tricuspid annulus circumference (TAC) 


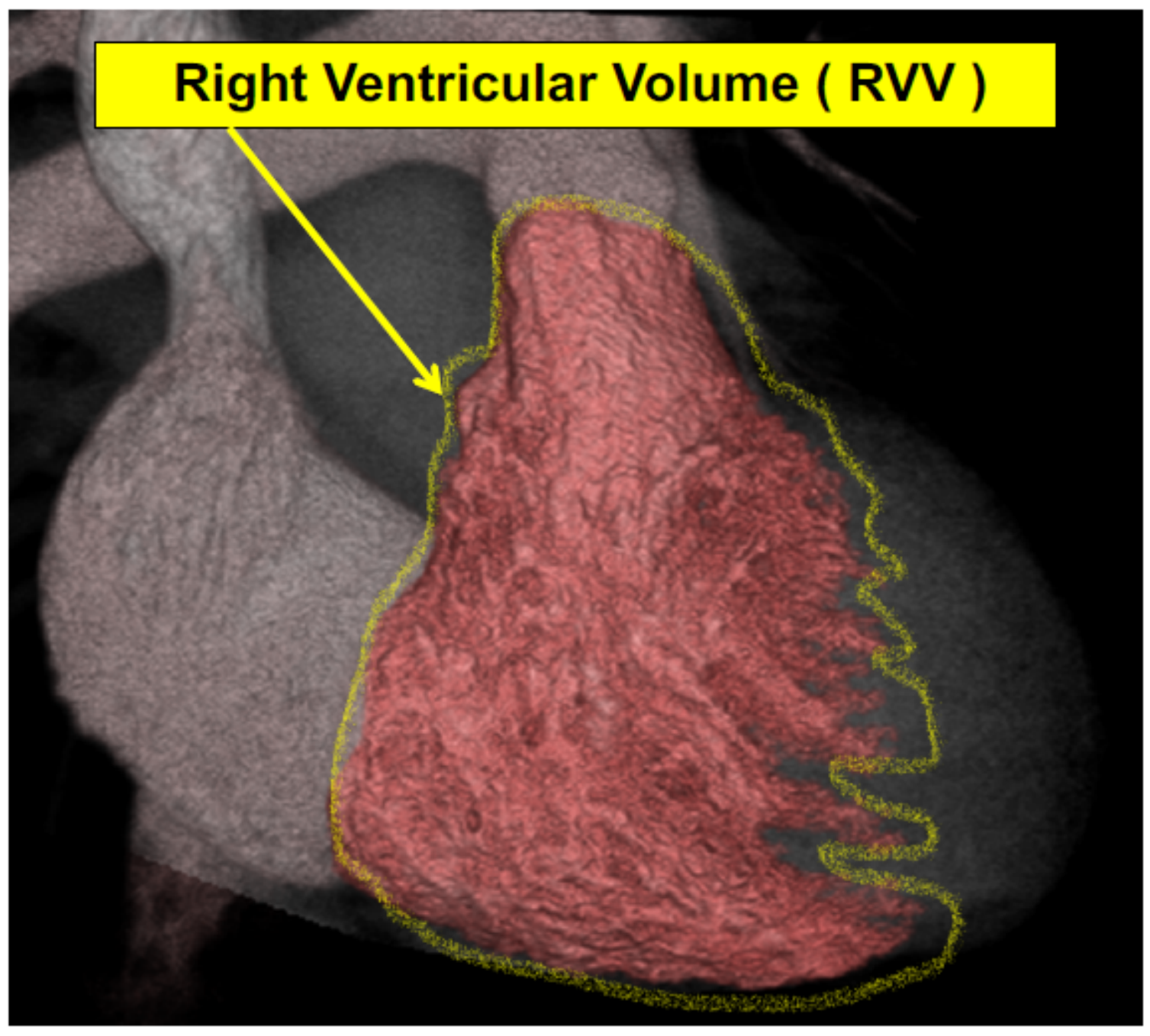

Figure 2

Right ventricular volume (RVV)
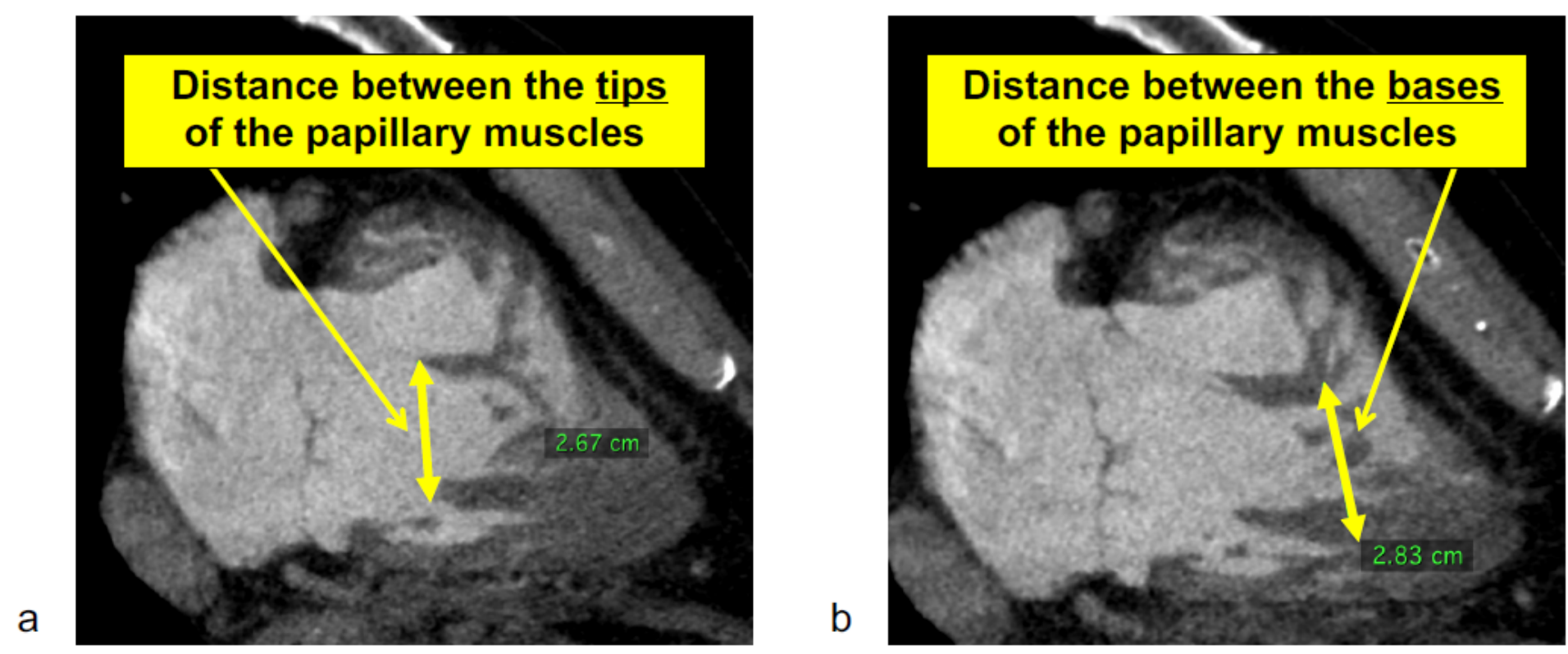
Figure 3

(a) Distance between the tips of the papillary muscles, (b) Distance between the bases of the papillary muscles
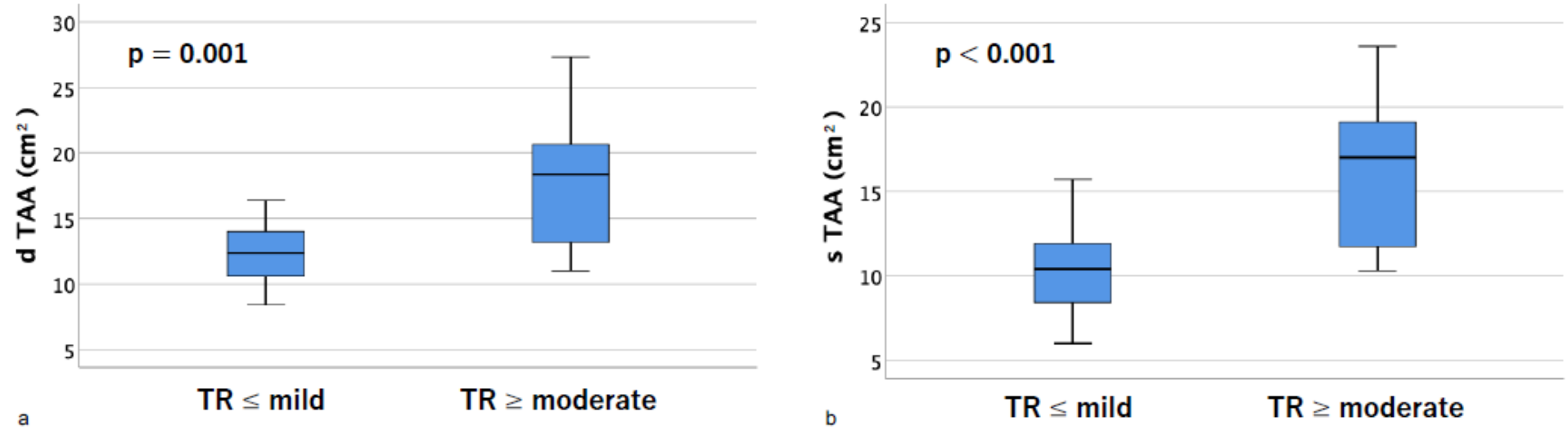

\section{Figure 4}

Box-and-whisker plot of diastolic (a) and systolic (b) tricuspid annulus area (TAA)
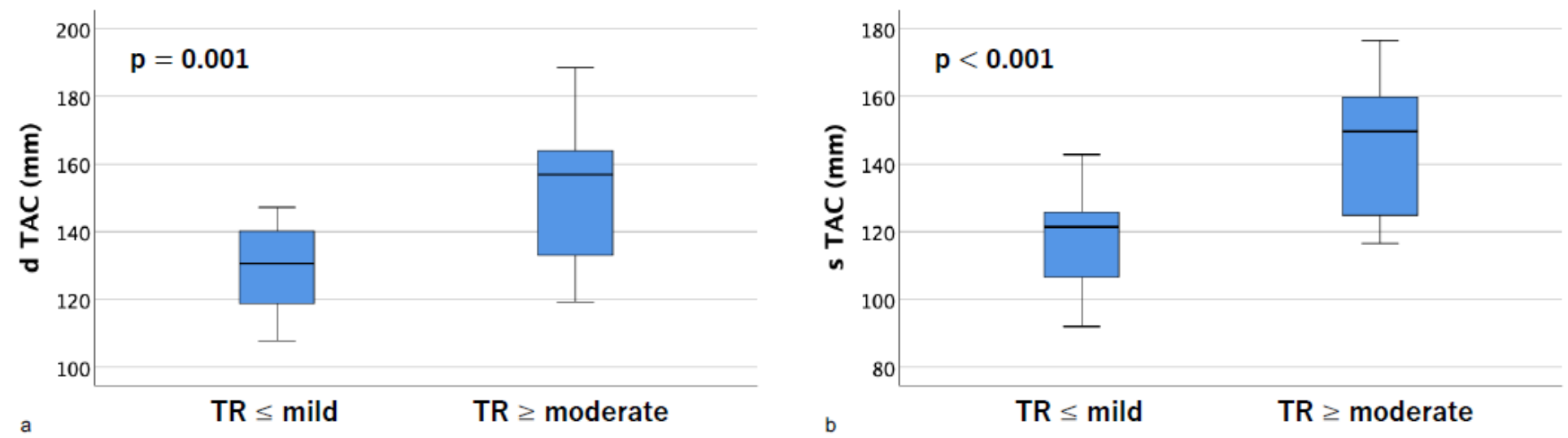

\section{Figure 5}

Box-and-whisker plot of diastolic (a) and systolic (b) tricuspid annulus circumference (TAC)
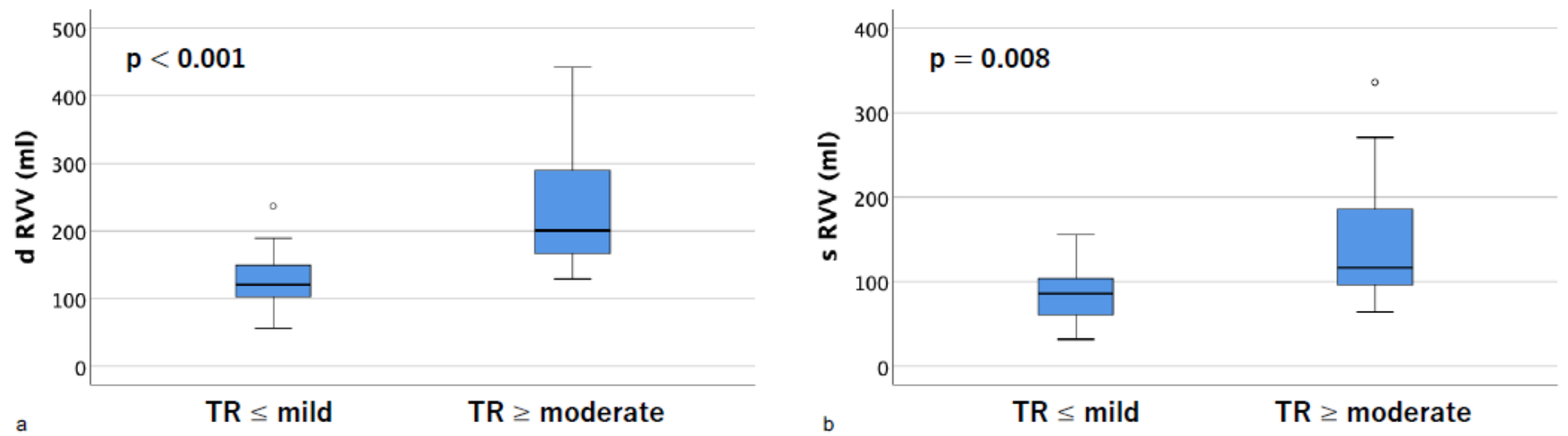
Figure 6

Box-and-whisker plot of diastolic (a) and systolic (b) right ventricular volumes (RVV)
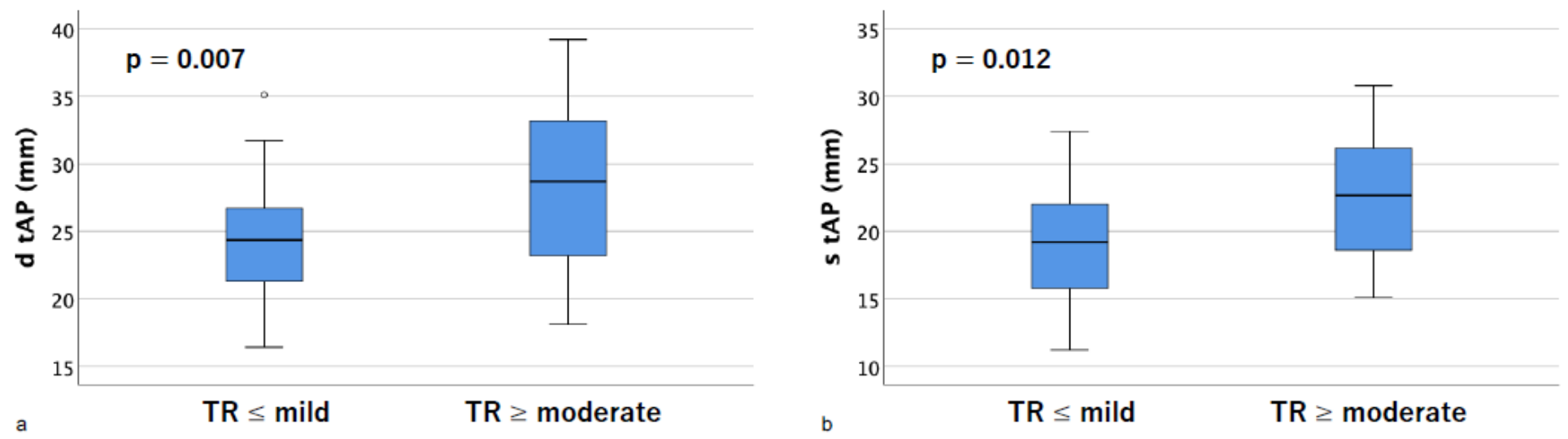

Figure 7

Box-and-whisker plot of diastolic (a) and systolic (b) distances between the tips of the anterior and posterior papillary muscles (tAP)
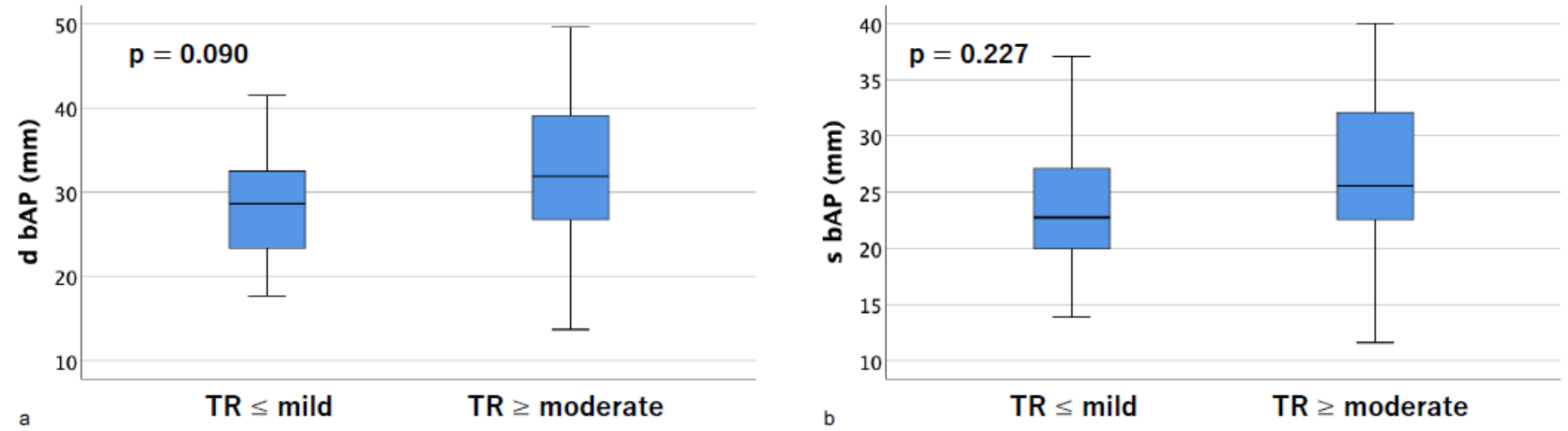

\section{Figure 8}

Box-and-whisker plot of diastolic (a) and systolic (b) distances between the bases of the anterior and posterior papillary muscles (bAP) 